Objectives: To understand the efficacy of EMDR in children and adolescent that experienced trauma. Materials and Methods: Non-systematic review using Pubmed with the keywords: "EMDR in children" "EMDR in adolescents"

\section{What is EMDR?}

Eye Movement Desensitization and Reprocessing (EMDR) is a fast and noninvasive psychosocial treatment for posttraumatic stress complaints. 4

In EMDR the individual focuses on a disturbing image, memory, emotion or cognition associated with the trauma (target) while the therapist initiates rapid voluntary eye movements.

These saccadic eye movements are elicited mainly to alleviate negative cognition, negative emotion, and unpleasant physical sensations associated with a traumatic memory and to reinforce positive cognition. 5

\section{$\underline{\text { Early life trauma }}$}
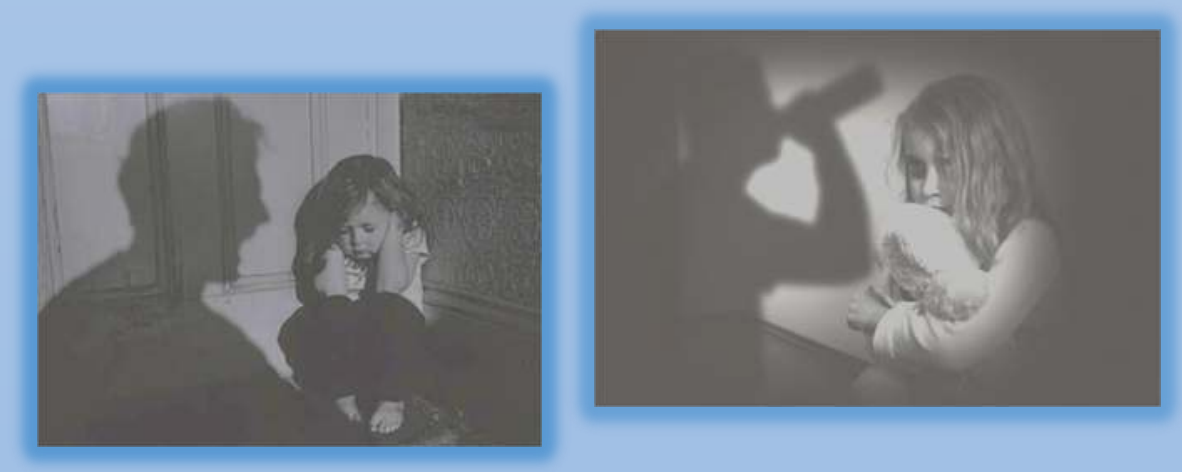

Epidemiological studies show that the highest risk period for exposure to many potentially traumatic events is during adolescence.5 Children can also suffer highly stressful events like domestic violence, physical and/or sexual abuse, neglect, or chronic illnesses which can contribute to the development of a posttraumatic stress disorder (PTSD) with or without a comorbid psychiatric disorder. The exposure to early life stressful events can be considered as a major risk factor for mental disorders, hence a rapid trauma orientated intervention is essential to prevent these adverse long term effects. This is especially true in children and adolescents due to their greater vulnerability during brain maturation. 5

\section{EMDR and its use in trauma}

Currently, trauma-focused cognitive behavioral therapy (TF-CBT) is the most acknowledged, evidence- based treatment for PTSD in children. 4

\section{Compared to TF-CBT, EMDR does not involve:}

(a) detailed descriptions of the event,

(b) direct challenging of beliefs,

(c) extended exposure

(d) homework.

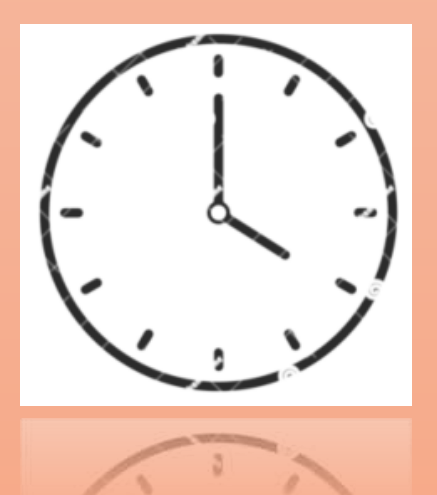

Therefore, EMDR is faster (often $<8$ sessions at $60 \mathrm{~min}$ ) than traditional TFCBT (8-12 sessions at $90 \mathrm{~min}$ ), cheaper and more efficient. 4

\section{Components of the EMDR protocol include:}

* Psychoeducation about the trauma and therapy

* Preparation of the target memory

* Desensitization of the memory

* Identification and processing of body sensations

* Re-evaluation of the target

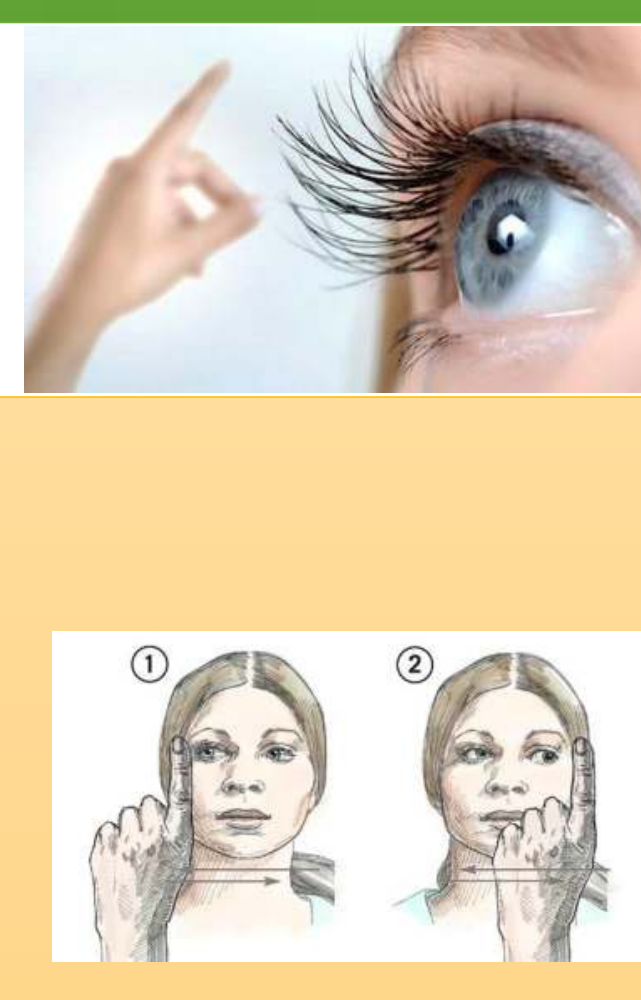

Theoretical framework - The working memory theory

The rationale is that humans have limited working memory capacity and engaging in dual-attention tasks therefore reduces the vividness and emotional intensity of memories. 4

\section{Evidence}

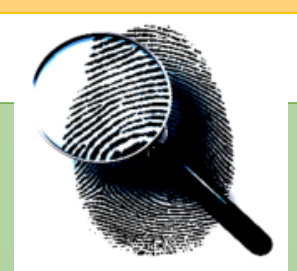

* When the children treated with EMDR were compared to the children treated with established trauma treatments (CBT), EMDR adds a small but significant incremental value. 6

* According to a recent meta-analysis, EMDR is better than CBT in reducing posttraumatic symptoms and anxiety. 3 But there was no difference reported in reducing depression. Participants treated with EMDR had better alleviation of post-traumatic symptoms as compared to participants treated with CBT $(p=0.006)$. However, the superiority of EMDR at three months follow-up was not evident. The EMDR also had a statistically significant superiority over CBT $(p=0.005)$ in alleviating anxiety. 3

* In other studies there was a small effect favoring CBT over EMDR, play therapy and supportive therapies.2

Non-CBT treatments (including EMDR) when compared to CBT have received less research attention, and the few existing studies that met review criteria tended to have more methodological weaknesses (e.g., small sample sizes, nonrandomized designs). 1

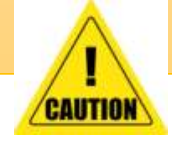

\section{Conclusions:}

The effectiveness of EMDR on PTSD in adults has been demonstrated in various reviews and in a meta-analysis. 4 However, the research about EMDR is still in its infancy, and more research is needed to understand better its mechanism of action and the underlying neural mechanism. More studies are also needed to confirm the preliminary results about the effectiveness of this psychotherapeutic approach in children and adolescents suffering from PTSD.4

\title{
REFERENCES
}

1- Dorsey, Shannon, and Katie McLaughlin. "Evidence Base Update for Psychosocial Treatments for Children and Adolescents Exposed to Traumatic Events." Taylor \& Francis, 2017

2- Gillies, Donna, et al. "Psychological Therapies for Children and Adolescents Exposed to Trauma - Gillies, D - 2016: Cochrane Library." Cochrane Database of Systematic Reviews, John Wiley \& Sons, Ltd, 11 Oct. 2016

3- Khan, Ali, et al. "Cognitive Behavioral Therapy versus Eye Movement Desensitization and Reprocessing in Patients with Post-Traumatic Stress Disorder: Systematic Review and Meta-Analysis of Randomized Clinical Trials." Cureus, 4 Sept. 2018.

4- Meentken, Maya G, et al. "Eye Movement Desensitization and Reprocessing (EMDR) in Children and Adolescents with Subthreshold PTSD after Medically Related Trauma: Design of a Randomized Controlled Trial." PubFacts, Eur J Psychotraumatol, June 2018

5- Moreno-Alcázar, Ana, et al. "Efficacy of Eye Movement Desensitization and Reprocessing in Children and Adolescent with Post-Traumatic Stress Disorder: A MetaAnalysis of Randomized Controlled Trials." Frontiers in Psychology, Frontiers Media S.A., 10 Oct. 2017

6- Rodenburg, Roos, et al. "Efficacy of EMDR in Children: A Meta-Analysis." Clinical Psychology Review, Pergamon, 24 June 2009 